\title{
THE DILATATION OF BEURLING-AHLFORS EXTENSIONS OF QUASISYMMETRIC FUNCTIONS
}

\author{
MATTI LEHTINEN
}

1. Introduction. The standard construction for a quasiconformal extension of a real quasisymmetric function $h$ is the one introduced by Beurling and Ahlfors [1]. They proved that a $\varrho$-quasisymmetric $h$ has a $\varrho^{2}$-quasiconformal extension to the upper half-plane $H$. A careful examination of Beurling's and Ahlfors's estimation shows that $\varrho^{2}$ can be replaced by $\varrho^{3 / 2}$ for $\varrho$ close to one and by $3 \varrho^{2} / 4$ for large $\varrho$ [4]. T. Reed [6] has given the bound $8 \varrho$, which is better than the previous ones for large $\varrho$. The bound $2 \varrho$ has been announced by Wan-cai Lai [3], but the correctness of his proof has been doubted (cf. [7]). In this note we shall establish the dilatation bound $2 \varrho$, using elementary methods different from those of Lai.

It is well known that to every quasisymmetric $h$ there exist one or more extremal extensions, i.e., quasiconformal maps $f: H \rightarrow H$ with smallest maximal dilation among those quasiconformal self-maps of $H$ which agree with $h$ on the boundary. In general, the explicit form of the extremal extensions of a given $h$ is not known. If $h$ is $\varrho$-quassymmetric with $\varrho$ close to one, $h$ has a Beurling-Ahlfors extension with maximal dilatation also close to one. However, K. Strebel [9] has shown by a simple normal family argument that no Beurling-Ahlfors extension of the $\varrho$-quasisymmetric function $h, h(x)=x$ for $x<0, h(x)=\varrho x, \varrho>1$, for $x \geqq 0$, can have maximal dilatation arbitrarily close to the dilatation of the corresponding extremal (which is explicitly known in this case).

In this note, we shall complement Strebel's result by showing that if $\varrho(h)$ is the the smallest number $\varrho$ for which $h$ is $\varrho$-quasisymmetric, then all Beurling-Ahlfors extensions of $h$ have maximal dilatation at least $\varrho(h)$. As a consequence of this one sees that Beurling-Ahlfors extensions can have considerably larger dilatation than the corresponding extremals. Consider, for instance, the affine stretching $x+i y_{\mapsto} \mapsto K x+i y$ of a square with sides parallel to the coordinate axes. If the map is lifted with the aid of two conformal transformations to a $K$-quasiconformal map $f: H \rightarrow H$, the boundary function $h=f \mid R^{1}$ satisfies $\varrho(h)=\lambda(K)=(1 / 16) \exp (\pi K)$ $-1 / 2+o(1)[5$, p. 81].

2. The upper bound. Given an increasing $\varrho$-quasisymmetric $h: R^{1} \rightarrow R^{1}$ and 
a constant $r>0$, the Beurling-Ahlfors extension $f_{h, r}: H \rightarrow H$ is constructed as follows: for $z=x+i y \in H$, set

and

$$
y \alpha(z)=\int_{0}^{y} h(x+t) d t, \quad y \beta(z)=\int_{-y}^{0} h(x+t) d t,
$$

$$
2 f_{h, r}(z)=\alpha(z)+\beta(z)+i r(\alpha(z)-\beta(z)) .
$$

Since linear transformations do not affect the property of being $\varrho$-quasisymmetric or the dilatation of a quasiconformal mapping, we may make certain simplifying assumptions when estimating the dilatation quotient of $f_{h, r}$ at an arbitrary $z$. First, we may suppose that $h$ is normalized, i.e., satisfies $h(0)=0$ and $h(1)=1$, and secondly, we may restrict ourselves to the point $z=i$. These conventions are obeyed in the rest of this section.

The dilatation quotient $D$ of $f_{h, r}$ at $i$ satisfies

$$
2 r(\zeta+\eta)\left(D+D^{-1}\right)=\left(1+r^{2}\right)\left(\zeta\left(1+\xi^{2}\right)+\zeta^{-1}\left(1+\eta^{2}\right)\right)+2(1-\zeta \eta)\left(1-r^{2}\right),
$$

where $\zeta=\alpha_{x} / \beta_{x}, \xi=\alpha_{y} / \alpha_{x}, \eta=\beta_{y} / \beta_{x}$. Since $h$ is normalized, one easily gets $\alpha_{x}(i)=1$, $\beta_{x}(i)=-h(-1), \alpha_{y}(i)=1-\int_{0}^{1} h(t) d t$ and $\beta_{y}(i)=h(-1)-\int_{-1}^{0} h(t) d t$. The $\varrho$-quasisymmetry of $h$ immediately yields $\varrho^{-1} \leqq \zeta \leqq \varrho$. By a lemma of Beurling and Ahlfors [1, p. 137],

$$
\lambda \leqq \int_{0}^{1} h(t) d t \leqq \mu
$$

where $\lambda=(1+\varrho)^{-1}$ and $\mu=\varrho \lambda$. It follows that $\xi$ and $\eta$ both lie in the interval $[\lambda, \mu]$.

Remark. The bounds in (2) are not the best possible. Equality in, say, the right hand side of (2) holds for the non-quasisymmetric majorant for normalized Q-quasiconformal functions introduced by R. Salem [8] and later studied by K. Goldberg [2]. By applying (2) repeatedly to properly chosen subintervals of $[0,1]$ one obtains slightly sharper bounds. For our purposes the improvement is negligible, and will not be taken into account.

The range of $(\xi, \eta, \zeta)$ is further restricted by the following

Lemma. If $h$ is a normalized Q-quasisymmetric function, then

$$
\int_{0}^{1} h(t) d t \leqq(2 \varrho+1) \int_{-1}^{0} h(t) d t-2 \varrho h(-1)
$$

and

$$
\int_{0}^{1} h(t) d t \leqq-\varrho \int_{-1}^{0} h(t) d t
$$

Proof. The $\varrho$-quasisymmetry of $h$ implies

$$
h(t)-h((t-1) / 2) \leqq \varrho(h((t-1) / 2)-h(-1))
$$


for all $t \in[-1,1]$, and (3) follows by integration. Also, since $h$ is normalized, $h(t) \leqq-\varrho h(-t)$ for all $t \in[0,1]$, and (4) follows.

When we use the notation adopted above, (3) and (4) become

$$
\zeta \leqq((2 \varrho+1) \eta-1) /(1-\xi)
$$

and

$$
\zeta \leqq \varrho(1-\eta) /(1-\xi)
$$

When estimating $D$ from above, we simplify computations by taking $r=1$. This is motivated by computations in [4], which indicate that for large $\varrho, f_{h, r}$ with $r$ close to 1 are the best choices for small maximal dilatation.

Theorem 1. The maximal dilatation of the Beurling-Ahlfors extension $f_{h, 1}$ of a @-quasisymmetric function $h$ is at most $2 \varrho$.

Proof. It suffices to prove $D \leqq 2 \varrho$, where $D$ is the dilatation quotient at $i$ of $f_{h, 1}$ for a normalized $\varrho$-quasisymmetric $h$. By (1),

$$
D+D^{-1}=\left(\zeta\left(1+\zeta^{2}\right)+\zeta^{-1}\left(1+\eta^{2}\right)\right) /(\xi+\eta)=F(\xi, \eta, \zeta)
$$

Since we may, if need be, replace $h$ by $g, g(x)=h(-x) / h(-1)$, we may assume $\zeta \geqq 1$. With this restriction, $F$ is maximized for fixed $(\xi, \eta)$ either at $\zeta=1$ or at the largest possible value of $\zeta$. The first alternative can take place only for $\xi \leqq \eta$. In this case we have

$$
F_{\eta}(\xi, \eta, 1)=\left(\eta^{2}+2 \xi \eta-\xi^{2}-2\right) /(\xi+\eta)^{2} .
$$

The nominator is a quadratic polynomial in $\eta$, and it is negative for $\eta=0$ and $\eta=1$. Consequently,

$$
F(\xi, \eta, 1) \leqq F(\xi, \xi, 1)=\xi+\xi^{-1} \leqq \lambda+\lambda^{-1}
$$

for $\lambda \leqq \zeta \leqq \eta$, and $D \leqq \lambda^{-1}=\varrho+1 \leqq 2 \varrho$.

To estimate $F$ for $\zeta$ large, we divide the square $\{(\xi, \eta) \mid \lambda \leqq \xi \leqq \mu, \lambda \leqq \eta \leqq \mu\}$ into four domains $T_{1}, T_{1}^{\prime}, T_{2}$ and $T_{3} . \quad T_{1}$ is the triangle with vertices $(\mu, \lambda),(\mu, \mu)$ and $(v, v)$, where $v=(\varrho+1) /(3 \varrho+1), T_{1}^{\prime}$ is $T_{1}$ reflected over $\xi=\eta, T_{2}$ is the triangle with vertices $(\lambda, v),(v, v)$ and $(\lambda, \mu)$, and $T_{3}$ is the trapezoid with vertices $(\lambda, \lambda)$, $(\mu, \lambda),(v, v)$ and $(\lambda, v)$. If $(\xi, \eta) \in T_{1}, D+D^{-1} \leqq F(\xi, \eta, \varrho)$, and we can compute

$$
\varrho F_{\eta}(\xi, \eta, \varrho)=\left(2 \eta^{2}+2 \xi \eta-\left(\varrho^{2} \xi^{2}+\varrho^{2}+1\right)\right) /(\xi+\eta)^{2} .
$$

Since $F_{\eta}(\xi, 0, \varrho)<0$ and $F_{\eta}$ can change sign at most once for $\eta>0$, the maxima of $F(\xi, \eta, \varrho)$ in $\bar{T}_{1}$ are attained on the lines $L_{1}: \xi=\eta$ or $L_{2}: \varrho \xi+(2 \varrho+1) \eta=\varrho+1$. Since $F(\xi, \xi, \varrho)=\left(\zeta+\xi^{-1}\right)\left(\varrho+\varrho^{-1}\right) / 2$ the condition $F(\xi, \xi, \varrho) \leqq F(v, v, \varrho)$ holds on $L_{1} \cap \bar{T}_{1}$. A lengthy but elementary computation shows that $F(v, v, \varrho) \leqq 2 \varrho+$ $(2 \varrho)^{-1}$ is true for $\varrho \geqq 1$. On $L_{2}$ we estimate $F_{1}(\eta)=F\left(1+\varrho^{-1}-\left(2+\varrho^{-1}\right) \eta, \eta, \varrho\right)$. By computation,

$$
(1-\eta)^{2}(\varrho / 2 \mu) F_{1}^{\prime}(\eta)=-\left(2 \varrho^{2}+2 \varrho+1\right) \eta^{2}+\left(4 \varrho^{2}+4 \varrho+2\right) \eta-\left(\varrho^{2}+2 \varrho\right) .
$$


Since $F_{1}^{\prime}(0)<0, F_{1}^{\prime}(1)>0, F_{1}$ is maximized on $L_{2} \cap \bar{T}_{1}$ either at $\eta=v$ or at $\eta=\lambda$. The former case has already been treated, and a direct computation shows that $F_{1}(\lambda)=F(\mu, \lambda, \varrho)$ is at most $2 \varrho+(2 \varrho)^{-1}$ if $\varrho \geqq 1$.

Since $\zeta \geqq 1, \quad F(\xi, \eta, \varrho) \leqq F(\eta, \xi, \varrho)$ for $\xi \leqq \eta$. Hence the estimation of $F(\xi, \eta, \varrho)$ in $T_{1}^{\prime}$ reduces to the work done in $T_{1}$.

Next consider $(\xi, \eta) \in T_{2}$. By (6),

By computation,

$$
F(\xi, \eta, \zeta) \leqq F(\xi, \eta, \varrho(1-\eta) /(1-\xi))=F_{2}(\xi, \eta) .
$$

$$
\left(F_{2}\right)_{\eta}(\xi, \eta)=-\frac{\varrho\left(1+\xi^{2}\right)(1+\xi)}{1-\xi}+\frac{(1-\xi)^{2}\left(\eta^{2}+2 \frac{1+\xi}{1-\xi} \eta-1\right)}{\varrho(1-\eta)^{2}},
$$

and we see that $\left(F_{2}\right)_{\eta}(\xi, 0)<0$ and $\left(F_{2}\right)_{\eta}$ can change sign at most once for fixed $\xi$. It follows that the maximum of $F_{2}$ is attained either on $\bar{T}_{1}^{\prime} \cap \bar{T}_{2}$ or on $\eta=v$. The former possibility has already been considered. Reasoning exactly as above, we see that $F_{2}(\xi, v)$ is maximized either at $\xi=v$ or at $\xi=\lambda$. By direct computation one verifies

$$
F_{2}(\lambda, v) \leqq 2 \varrho+(2 \varrho)^{-1} .
$$

To complete the proof, $(\xi, \eta) \in T_{3}$ has still to be considered. By (5), we have in this case

$$
F(\xi, \eta, \zeta) \leqq F(\xi, \eta,((2 \varrho+1) \eta-1) /(1-\xi))=F_{3}(\xi, \eta) .
$$

The proof that $F_{3}(\xi, \eta) \leqq 2 \varrho+(2 \varrho)^{-1}$ in $T_{3}$ is carried out as above: by the consideration of $\left(F_{3}\right)_{\xi}$ the possible maximal set is reduced to $\xi=\lambda$ and $\bar{T}_{1} \cap \bar{T}_{3}$, and on $\xi=\lambda$ one only has to take care of the points $\eta=\lambda$ and $\eta=v$.

Remark. As $\varrho \rightarrow \infty$, (7) is asymptotically true as an equality. The upper limit $2 \varrho$ cannot be improved without further restriction of the range of $(\xi, \eta, \zeta)$.

3. The lower bound. In deriving a condition relating the lower bound of maximal dilatations of $f_{h, r}$ to the deviation from symmetry of $h$, it is natural to consider the smallest $\varrho$ for which $h$ is $\varrho$-quasisymmetric. We denote such a $\varrho$ by $\varrho(h)$.

Theorem 2. Let $h: R^{1} \rightarrow R^{1}$ be quasisymmetric. Then the maximal dilatation of every $f_{h, r}$ is at least $\varrho(h)$.

Proof. For every $\varrho<\varrho(h)$ there are points $x, t \in R^{1}$ such that

$$
h(x+t)-h(x)=\varrho(h(x)-h(x-t)) .
$$

There is no loss of generality in assuming that $h$ is normalized and $h(-1)^{-1}=\varrho$. By (1), the dilatation quotient $D$ of $f_{h, r}$ at $i$ satisfies

where

$$
D+D^{-1}=a(\xi, \eta) r+b(\xi, \eta) r^{-1},
$$

$$
\begin{aligned}
& 2 \varrho(\xi+\eta) a(\xi, \eta)=(\varrho-1)^{2}+(\varrho \xi+\eta)^{2}, \\
& 2 \varrho(\xi+\eta)^{\prime} b(\xi, \eta)=(\varrho+1)^{2}+(\varrho \xi-\eta)^{2}
\end{aligned}
$$


It follows that for fixed $(\xi, \eta)$

$$
\left(D+D^{-1}\right)^{2} \geqq 4 a(\xi, \eta) b(\xi, \eta)=F_{4}(\xi, \eta) .
$$

Since $h$ is $\varrho(h)$-quasisymmetric, (5) and (6) imply that the distance of $(\xi, \eta)$ from $T_{1}$ can be made arbitrarily small if $\varrho$ is chosen close to $\varrho(h)$. Considering $\left(F_{4}\right)_{\eta}$, one sees as before that

$$
\begin{gathered}
F_{4}(\xi, \eta) \geqq F_{4}(\xi, \xi)=\left((\varrho-1)^{4}+(\varrho+1)^{4}+\left(\varrho^{2}-1\right)^{2}\left(\xi^{2}+\xi^{-2}\right)\right) /\left(4 \varrho^{2}\right) \\
\geqq F_{4}(\mu, \mu)
\end{gathered}
$$

holds in $T_{1}$. By direct computation one verifies

$$
F_{4}(\mu, \mu) \geqq\left(\varrho+\varrho^{-1}\right)^{2}
$$

as a strict inequality for $\varrho>1$. Continuity of $F_{4}$ together with (8) yield $D+D^{-1}>$ $\varrho(h)+\varrho(h)^{-1}$ for the proper choice of $\varrho$.

\section{References}

[1] Beurling, A., and L. V. Ahlfors: The boundary correspondence under quasiconformal mappings. - Acta Math. 96, 1956, 125-142.

[2] Goldberg, K.: A new definition for quasisymmetric functions. - Michigan Math. J. 21, 1974, $49-62$.

[3] LaI, Wan-CaI: A theorem of Beurling and Ahlfors. - Acta Math. Sinica 22, 1979, 178-184 (Chinese).

[4] Lehtinen, M.: A real-analytic quasiconformal extension of a quasisymmetric function. - Ann. Acad. Sci. Fenn. Ser. A I Math. 3, 1977, 207-213.

[5] Lehto, O., and K. I. Virtanen: Quasiconformal mappings in the plane. - Springer-Verlag, Berlin-Heidelberg-New York, 1973.

[6] REED, T : Quasiconformal mappings with given boundary values. - Duke Math. J. 33, 1966, 459-464.

[7] ReICH, E.: Review 30020 in Math. Reviews 81m, 1981, 4947.

[8] SALEM, R.: On some singular monotonic functions which are strictly increasing. - Trans. Amer. Math. Soc. 53, 1943, 427-439.

[9] Strebel, K.: On the existence of extremal Teichmueller mappings. - J. Analyse Math. 30, 1976, $464-480$.

University of Helsinki

Department of Mathematics

SF-00100 Helsinki 10

Finland

Received 25 November 1982 